\title{
The Characteristics of Action Potentials in Primo Vessels and the Effects of Acetylcholine Injection to the Action Potentials
}

\author{
Seong Jin Cho, ${ }^{1}$ Jaekwan Lim, ${ }^{2}$ Sun Hee Yeon,, ${ }^{1}$. Sang Kwon, ${ }^{1}$ Kwang-Ho Choi, \\ Sun-Mi Choi, ${ }^{1}$ and Yeon-Hee Ryu ${ }^{1}$ \\ ${ }^{1}$ Acupuncture, Moxibustion, and Meridian Research Group, Medical Research Division, Korea Institute of Oriental Medicine, \\ Daejeon 305-811, Republic of Korea \\ ${ }^{2}$ Advanced Institutes of Convergence Technology, Seoul National University, Suwon, Republic of Korea
}

Correspondence should be addressed to Yeon-Hee Ryu; yhryu@kiom.re.kr

Received 18 February 2013; Revised 16 May 2013; Accepted 20 May 2013

Academic Editor: Pradeep Visen

Copyright (C) 2013 Seong Jin Cho et al. This is an open access article distributed under the Creative Commons Attribution License, which permits unrestricted use, distribution, and reproduction in any medium, provided the original work is properly cited.

\begin{abstract}
In a previous study, we found that Primo vessels generate different action potentials in smooth muscles, but this study compared the pulse shape to distinguish the two tissues. Thus, a more sophisticated extracellular experiment was performed in this study using an acetylcholine injection; we then observed changes in the amplitude, FWHM (full width at half maximum), and period to explore Primo vessel function. A third type of pulse was recorded for Primo vessels. We observed fast depolarizing and repolarizing phases for this pulse. Further, its FWHM was $30 \mathrm{~ms}$ between smooth muscles and neurons. Acetylcholine affected only the period. The amplitude and FWHM were consistent after injection. Primo-vessels generated action potentials at twice the frequency after injection. From the results, we speculate that Primo-vessels perform a role in transferring signals in a different manner, which may be relevant for acupuncture treatment.
\end{abstract}

\section{Introduction}

Over the last decade Primo vascular system has attracted interests among researchers both anatomically and histologically. However, it is still unclear what functions Primo tissues do. Some researchers have found Primo tissues in organ surface, lymphatic vessels, cardiac vascular vessels, and brain. These findings have led them to consider Primo vascular system another circulating system [1-4]. Moreover, Soh claimed that Primo nodes and Primo-vessels were related to acupuncture points and Primo vascular system might be an extension of meridians [5]. Park started to measure action potentials in Primo-node for the first time in 2000s. He measured resting and spontaneous potentials and observed the affection of drugs [6]. More researchers have studied Primo-vessel electrophysiological characteristics after this study, and they have found bioelectrical signals from Primo-vessels and lymphatic vessels were different. However, the results have not been sufficient to clarify the functions $[7,8]$. In our previous study on Primo-vessel action potentials, we found that two types of pulses were generated that differed from the pacemaker pulse for smooth muscles in the small intestine. This result indicates that Primo-vessels have a distinct function from smooth muscles. However, it was difficult to determine what role Primo-vessels played because we only compared pulse shape to distinguish Primo-vessels and smooth muscles [9]. The action potentials from the different tissues have distinct pulse components, such as amplitude, duration, and frequency. Further, neurotransmitters can affect these components [1013]. Thus, the pulse components must be analyzed, and the reaction of tissues to neurotransmitters must be measured. In this study, we analyzed the action potentials from Primovessels and observed the effects of acetylcholine on the pulse component.

\section{Materials and Methods}

2.1. Animals and Tissue Preparation. Male 7-week-old Sprague-Dawley rats weighing $250-320 \mathrm{~g}$ were used. The rats 
were anesthetized with a $1.5 \mathrm{~g} / \mathrm{kg}$ urethane $\left(\mathrm{C}_{3} \mathrm{H}_{7} \mathrm{NO}_{2}\right)$ injection into the femoral region, and the entire surgical procedure was performed with the anesthetized rat. A midline abdominal incision was generated, and the internal organs were exposed. The large intestine surface Primo-vessels were removed from the rats and placed on a Sylgard. We then added phosphate buffered saline (PBS, which contained (in mM) $137 \mathrm{NaCl}, 27 \mathrm{KCl}, 10 \mathrm{Na}_{2} \mathrm{HPO}_{4}$, and $2 \mathrm{KH}_{2} \mathrm{PO}_{4}$ ) to the sample.

2.2. Equipment. The tissue preparations were viewed under a microscope (SMZ1500, Nikon, Japan), and a Fiber-Lite supplied the light (MI-150, Dolan Jenner industries, MA, USA). A second microscope (SZ61, Olympus, Japan) and micromanipulator (MP-225, Sutter Instrument, CA, USA) were used to insert the electrode (TM33B01, World Precision Instruments, FL, USA) into the tissue. An Ag/AgCl electrode (EP1, World Precision Instruments, FL, USA) was used as the reference electrode. The electric signals were amplified by a primary amplifier that contained a head amplifier (Duo773, World Precision Instruments, FL, USA) and acquired by using a data acquisition system (PowerLab/16SP, ADInstruments, CO, USA). A data acquisition program (LabChart 6, ADInstruments, CO, USA) was used to record the data, and the data were analyzed using Microsoft Excel 2010 (Microsoft, WA, USA).

2.3. Extracellular Recording. An electrode was placed in the tissue on the Sylgard. The tissue was perfused with Krebs' solution at an approximate $5 \mathrm{~mL} / \mathrm{min}$ constant flow rate. The Krebs' solution contained (in mM) 10.10 D-glucose, 115.48 $\mathrm{NaCl}, 21.90 \mathrm{NaHCO}_{3}, 4.61 \mathrm{KCl}, 1.14 \mathrm{NaH}_{2} \mathrm{PO}_{4}, 2.50 \mathrm{CaCl}_{2}$, and $1.16 \mathrm{MgSO}_{4}$. This solution was at $\mathrm{pH} 7.4$ and $36^{\circ} \mathrm{C}$. The solution temperature in the organ bath was maintained at $36 \sim 38^{\circ} \mathrm{C}$. The electrical responses were amplified, low pass filtered $(50 \mathrm{~Hz})$, and recorded (time interval: $0.0005 \mathrm{sec}$ ) on a computer. The laboratory was isolated from electromagnetic noise by a Faraday cage. Acetylcholine (A6625-25G, Sigma Aldrich, MO, USA) was diluted 1000 times and injected into the solution 5 minutes after the action potential was detected. After recording the action potentials, H\&E staining was applied to the tissue.

2.4. Analysis. Two sections, 1 minute before and 1 minute after acetylcholine injection, were extracted in all samples. The amplitude, FWHM (full width at half maximum), and period, the time difference between one peak and the next peak, were calculated from the action potentials and averaged totally in each and compared by performing $t$-test.

\section{Results}

We tried to measure action potentials in 10 samples, and these samples were verified by comparing anatomical and histological characteristics with other studies. Figure 1 shows the stereomicroscopic image of a sample used in this study, and Figure 2 shows the H\&E staining result of the sample.

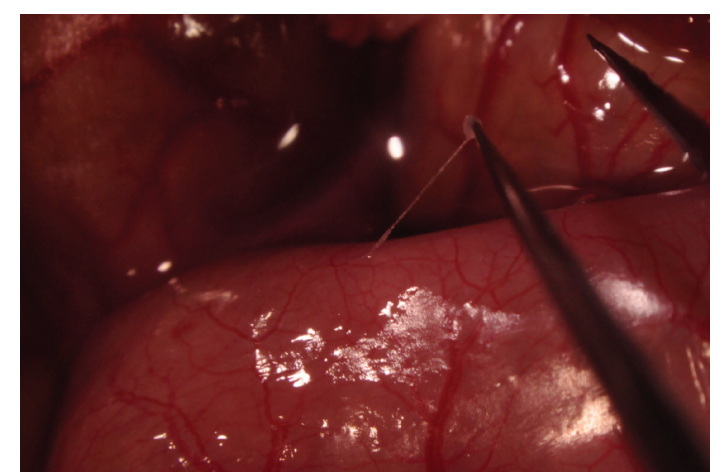

Figure 1: Stereomicroscopic image of Primo-vessel used in this study. This sample was taken from large intestine in SD rat. This sample had thread-like structure and milky-white color as the other studies reported.

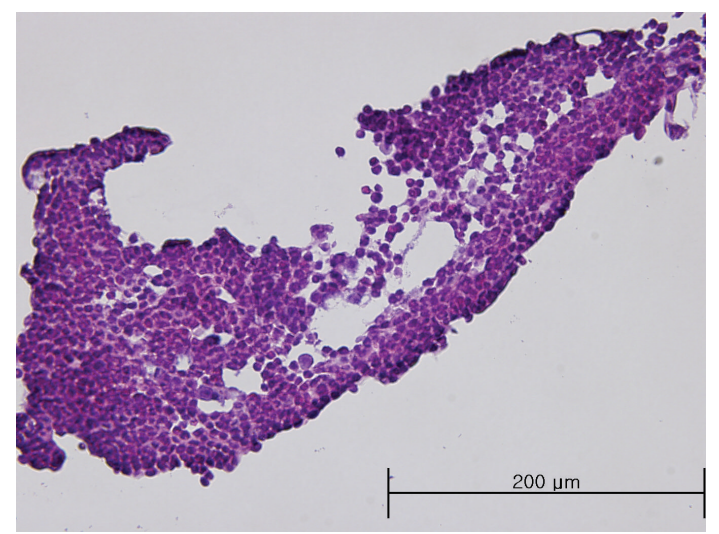

FIGURE 2: H\&E staining result of the sample shown Figure 1. It was the same histological characteristic of Primo-vessels with other studies that the tissue was filled with cells.

The anatomical structure and the histological feature were same with other studies $[14,15]$.

We measured spontaneous action potentials before and after acetylcholine injection in 3 samples. Figure 3 shows the representative action potentials generated from Primovessels in this study. The pulses had rapid depolarizing and repolarizing phases, such as the Type I pulse in our previous study. However, the difference from our previous study is that the action potentials were generated periodically both before and after acetylcholine injection.

For a detailed comparison of the action potentials from the before and after sections, the amplitude, FWHM, and period were calculated in each section. The pulses in before section had a $0.53 \pm 0.10 \mathrm{mV}$ mean amplitude, and the pulses in after section had a $0.59 \pm 0.10 \mathrm{mV}$ mean amplitude. The amplitude was slightly but not significantly increased after injection. The FWHM for the pulses was around $30 \mathrm{~ms}$ for both sections. However, there was a significant variation in the period. The Primo-vessels generated an action potential with a $2.234 \pm 0.442 \mathrm{~s}$ period before injection. After injection, the period decreased by half, $1.364 \pm 0.474$ (Table 1). Figure 4 
TABLE 1: The amplitude, FWHM and period for the Primo-vessel action potentials. The amplitude is the difference between the maximum and rest potentials. The FWHM is the time difference between two points that is equal to half of the amplitude. The period is the time difference between one peak and the next peak. The amplitude was slightly but not significantly increased after acetylcholine injection. The FWHM was not changed by acetylcholine. The period was decreased after injection.

\begin{tabular}{lccc}
\hline & Amplitude $(\mathrm{mV})$ & FWHM (ms) & Period (s) \\
\hline Before injection & $0.53 \pm 0.10$ & $31 \pm 7$ & $2.234 \pm 0.442$ \\
After injection & $0.59 \pm 0.10$ & $32 \pm 8$ & $1.364 \pm 0.474$ \\
\hline
\end{tabular}

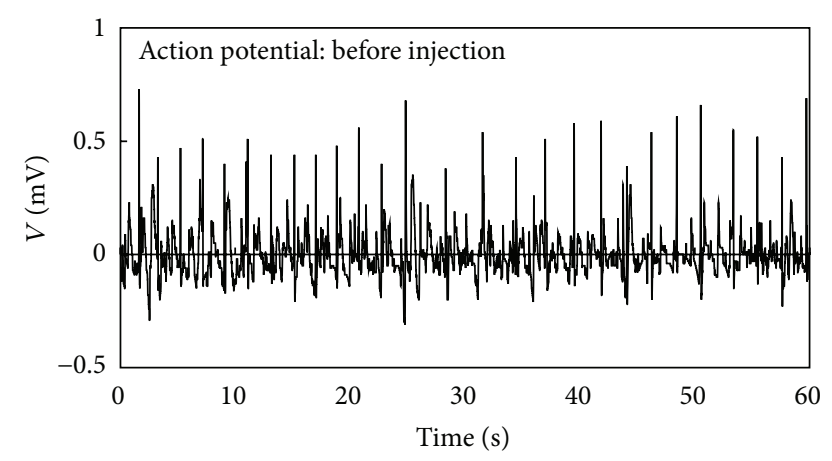

(a)

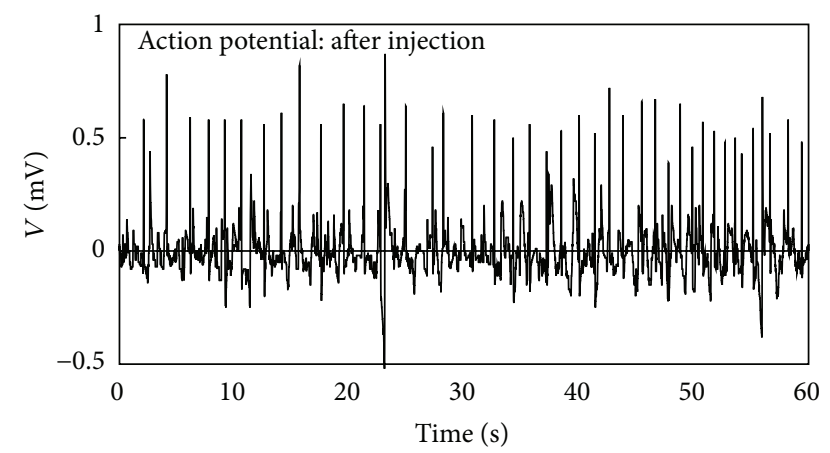

(b)

Figure 3: Representative Primo-vessel action potentials. (a) The action potentials for a section 1 minute before acetylcholine injection. (b) The action potentials for a section 1 minute after acetylcholine injection. The pulses rose and fell rapidly and were generated periodically in both sections.

shows a comparison of the amplitude, FWHM, and period for the before and after injection sections in all samples.

Further, we observed time variations for the period. Figure 5 shows the variation in the period for the time domain. This figure was gained from the same sample which was used in Figure 3. Primo-vessels generated action potentials with a $2 \mathrm{~s}$ period before acetylcholine injection, but the period decreased gradually to $1 \mathrm{~s}$.

\section{Discussion}

In this study, we analyzed action potential pulse components. Because of inaccuracy in the extracellular recording

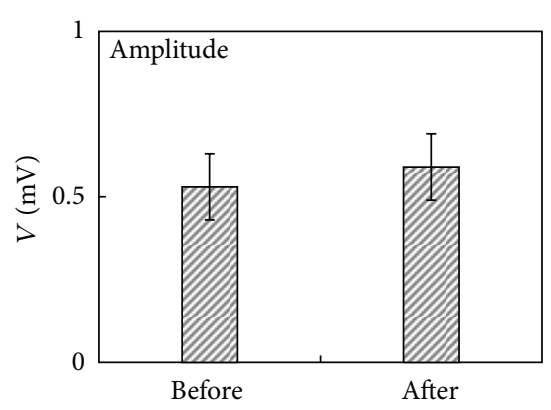

(a)

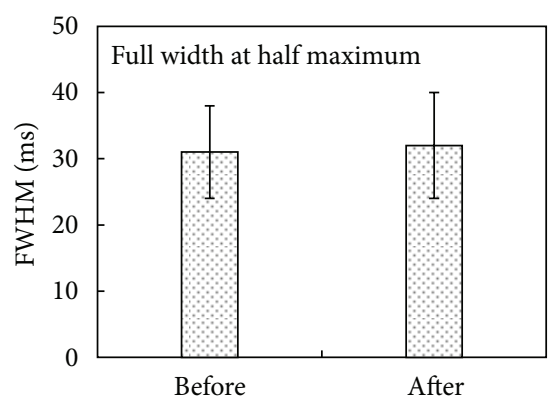

(b)

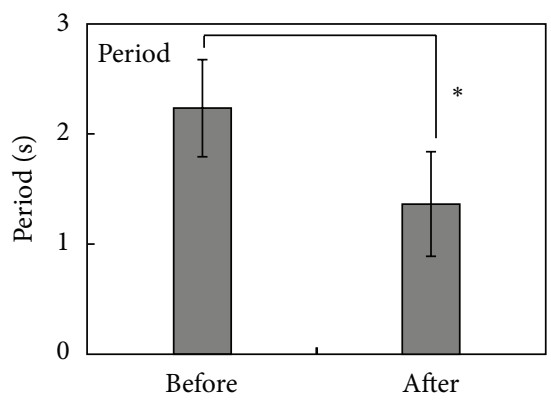

(c)

FIgURE 4: A comparison of the amplitude, FWHM, and period for sections before and after acetylcholine injection. (a) The amplitude was slightly but not significantly increased after injection. (b) The FWHM was maintained in both sections. (c) The period decreased after injection. Acetylcholine activated the Primo-vessels.

amplitudes, the amplitude absolute values had no meaning. However, we did observe an effect from acetylcholine injection by comparing the amplitude change before and after injection. The action potential amplitudes for Primo-vessels were slightly increased by approximately $13 \%$ after the acetylcholine injection. It is hard to assert that acetylcholine facilitates action potentials in Primo-vessels with larger amplitudes because the amplitude variation is in the error range. Previous studies have reported the same results; that is, acetylcholine had no effect on action potential amplitude. The pulses had approximately 30 ms FWHMs in this study, which is approximately one-tenth of the value reported in our previous study. These pulses do not belong to Type I or Type II. Thus, we categorized these pulses as Type III with fast depolarizing and repolarizing phases. The FWHM for these pulses was shorter than in smooth muscles from the small intestine 


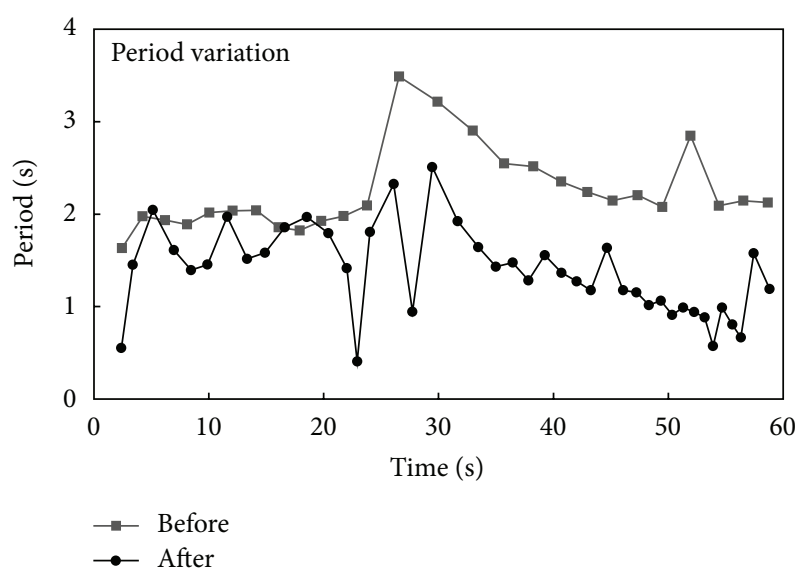

FIGURE 5: Representative time variation for period before and after injection. Under normal conditions, the pulses generated with a $2 \mathrm{~s}$ period. After acetylcholine injection, the period decreased gradually to half of the pre-injection value.

or lymphatic vessels $(\sim 500 \mathrm{~ms})$ and longer than in neurons ( 1 ms) [16]. The small intestine and lymphatic vessels generate an action potential to transfer materials. Neuron spikes are generated when neurons exchange electrical signals [17]. Primo-vessel has median values of its size and FWHM between smooth muscle and a neuron. This result indicates that Primo-vessels perform different functions in smooth muscles and neurons. We speculate that Primo-vessels transfer signals in distinct manners for neurons and do not directly move materials, such as through the small intestine and lymphatic vessels. Primo-vessels were considered the substances in acupuncture points and meridians [5]. Therefore, signals may contain information from acupuncture stimulation if Primo-vessels have such a role. The FWHM was maintained after the acetylcholine injection. Thus, acetylcholine did not affect the FWHM for the action potentials in Primovessels. This result is consistent with other studies involving acetylcholine.

However, there was a significant change in the period after acetylcholine injection. The period is the time interval between the two serial pulses. Primo-vessels generated Type III pulses with a $2.234 \mathrm{~s}$ period on average under normal condition. Acetylcholine decreased the period to $1.364 \mathrm{~s}$. Thus, acetylcholine enhanced Primo-vessels activation and generated action potentials more frequently. In Figure 5, the period was maintained as under normal conditions for $30 \mathrm{~s}$ and then gradually decreased to half the value of before section.

\section{Conclusion}

We found that Primo-vessels generated a third type of pulse with fast depolarization and repolarization. This type had a uniform amplitude, $30 \mathrm{~ms}$ FWHM, and $2 \mathrm{~s}$ period in normal condition. Primo-vessels' function was considered transferring electrical signals rather than carrying material due to the very short FWHM. Further, the amplitude and the FWHM of the action potentials from Primo-vessels were not changed by acetylcholine. However, acetylcholine decreased the period of the action potentials to $1 \mathrm{~s}$, half value of normal condition. It means that Primo vascular system can be controlled by acetylcholine. Further studies are necessary to clarify the functions of Primo-vessels and to establish the relation between Primo vascular system and meridian system.

\section{Conflict of Interests}

The authors declare that they have no conflict of interests.

\section{Acknowledgment}

This study was supported by grants from the Korea Institute of Oriental Medicine (K13290).

\section{References}

[1] H.-S. Shin, H.-M. Johng, B.-C. Lee et al., "Feulgen reaction study of novel threadlike structures (Bonghan ducts) on the surfaces of mammalian organs," Anatomical Record Part B, vol. 284, no. 1, pp. 35-40, 2005.

[2] B.-C. Lee and K.-S. Soh, "Contrast-enhancing optical method to observe a Bonghan duct floating inside a lymph vessel of a rabbit," Lymphology, vol. 41, no. 4, pp. 178-185, 2008.

[3] B.-C. Lee, H. B. Kim, B. Sung et al., "Network of endocardial vessels," Cardiology, vol. 118, no. 1, pp. 1-7, 2011.

[4] B.-C. Lee, S. Kim, and K.-S. Soh, "Novel anatomic structures in the brain and spinal cord of rabbit that may belong to the Bonghan system of potential acupuncture meridians," Journal of Acupuncture and Meridian Studies, vol. 1, no. 1, pp. 29-35, 2008.

[5] K.-S. Soh, "Bonghan circulatory system as an extension of acupuncture meridians," Journal of Acupuncture and Meridian Studies, vol. 2, no. 2, pp. 93-106, 2009.

[6] S. H. Park, Bioelectrical study of Bonghan system [Ph.D. thesis], Seoul National University, 2009.

[7] C.-J. Choi, J. H. Jung, K.-S. Soh, Y. H. Ryu, P. D. Ryu, and S.-H. Park, "Spontaneous action potential from threadlike structures on the surfaces of abdominal organs," Journal of the Korean Physical Society, vol. 58, no. 4, pp. 831-836, 2011.

[8] J.-H. Choi, C. J. Lim, T. H. Han, S. K. Lee, S. Y. Lee, and P. D. Ryu, "TEA-sensitive currents contribute to membrane potential of organ surface primo-node cells in rats," The Journal of membrane biology, vol. 239, no. 3, pp. 167-175, 2011.

[9] S.-J. Cho, S.-H. Lee, W. Zhang et al., "Mathematical distinction in action potential between primo-vessels and smooth muscle," Evidence-Based Complementary and Alternative Medicine, vol. 2012, Article ID 269397, 6 pages, 2012.

[10] Y. Kito and H. Suzuki, "Properties of pacemaker potentials recorded from myenteric interstitial cells of Cajal distributed in the mouse small intestine," Journal of Physiology, vol. 553, no. 3, pp. 803-818, 2003.

[11] H. Fukuta, Y. Kito, and H. Suzuki, "Spontaneous electrical activity and associated changes in calcium concentration in guinea-pig gastric smooth muscle," Journal of Physiology, vol. 540, no. 1, pp. 249-260, 2002.

[12] E. Terazima and M. Yoshino, "Modulatory action of acetylcholine on the $\mathrm{Na}^{+}$-dependent action potentials in Kenyon cells isolated from the mushroom body of the cricket brain," Journal of Insect Physiology, vol. 56, no. 12, pp. 1746-1754, 2010. 
[13] Y. Zhang, Y. Kakinuma, M. Ando et al., "Acetylcholine inhibits the hypoxia-induced reduction of connexin 43 protein in rat cardiomyocytes," Journal of Pharmacological Sciences, vol. 101, no. 3, pp. 214-222, 2006.

[14] J. S. Yoo, M. Hossein Ayati, H. B. Kim, W.-B. Zhang, and K.-S. Soh, "Characterization of the primo-vascular system in the abdominal cavity of lung cancer mouse model and its differences from the lymphatic system," PLoS ONE, vol. 5, no. 4, Article ID e9940, 2010.

[15] B. S. Kwon, C. M. Ha, S. Yu, B. C. Lee, J. Y. Ro, and S. Hwang, "Microscopic nodes and ducts inside lymphatics and on the surface of internal organs are rich in granulocytes and secretory granules," Cytokine, vol. 60, no. 2, pp. 587-592, 2012.

[16] K. Seetharaman, H. Namazi, and V. V. Kulsih, "Phase lagging model of brain response to external stimuli modeling of single action potential," Computers in BiologyandMedicine, vol. 42, pp. 857-862, 2012.

[17] H. Shen and W. Choe, "Spontaneous high-frequency action potential," Science China Life Sciences, vol. 54, no. 4, pp. 311-335, 2011. 


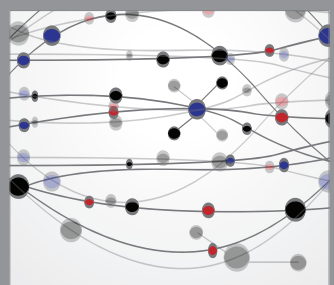

The Scientific World Journal
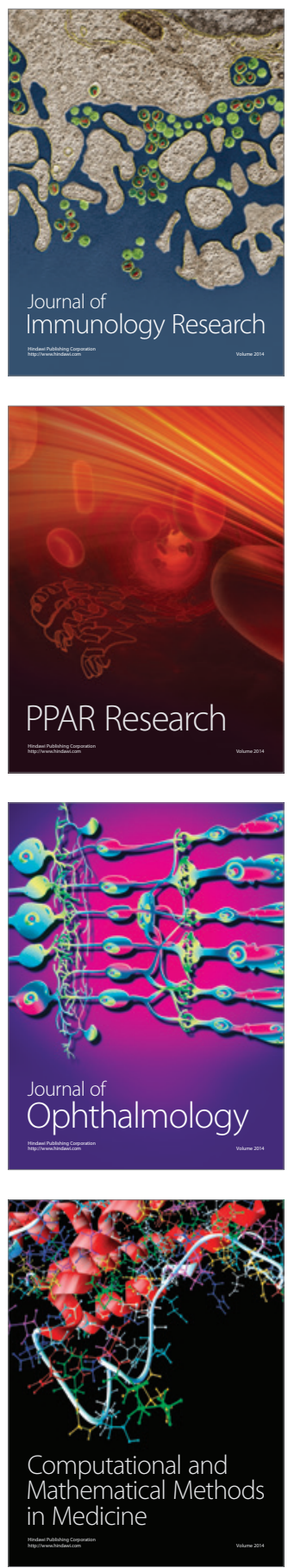

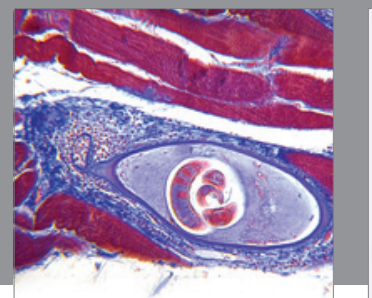

Gastroenterology

Research and Practice
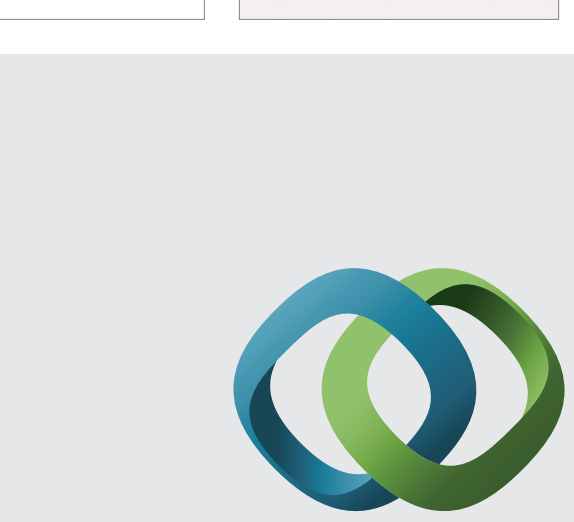

\section{Hindawi}

Submit your manuscripts at

http://www.hindawi.com
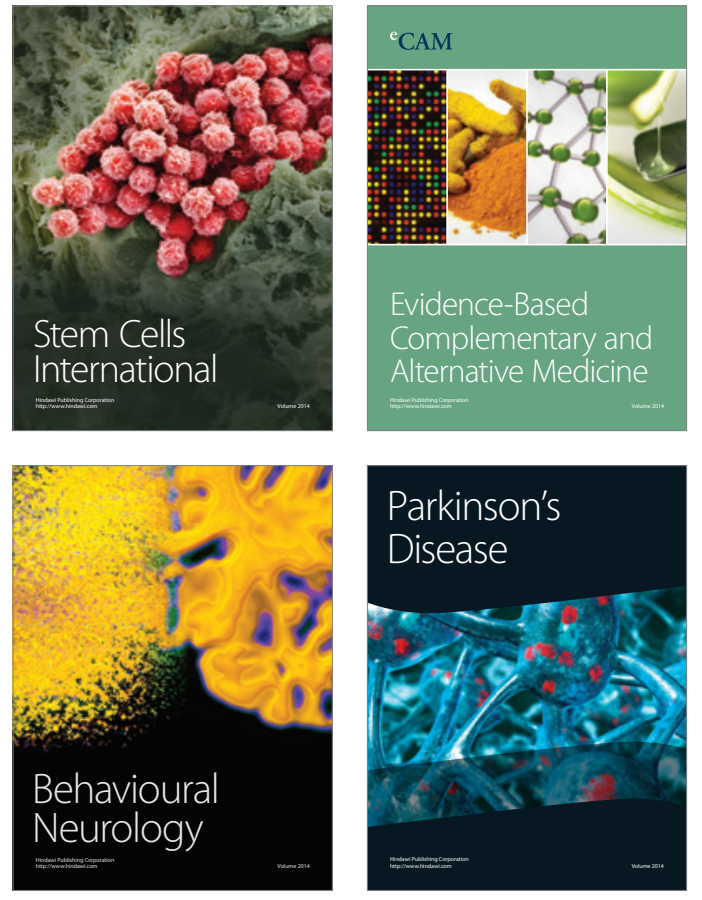
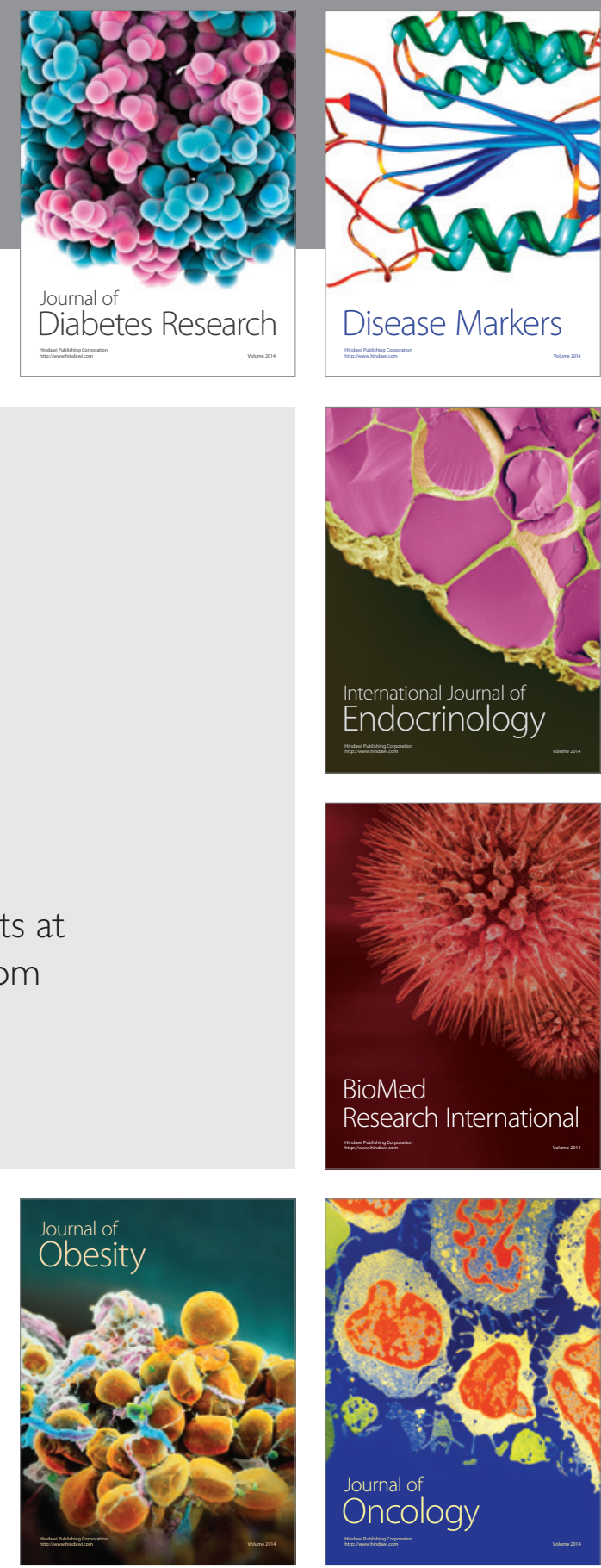

Disease Markers
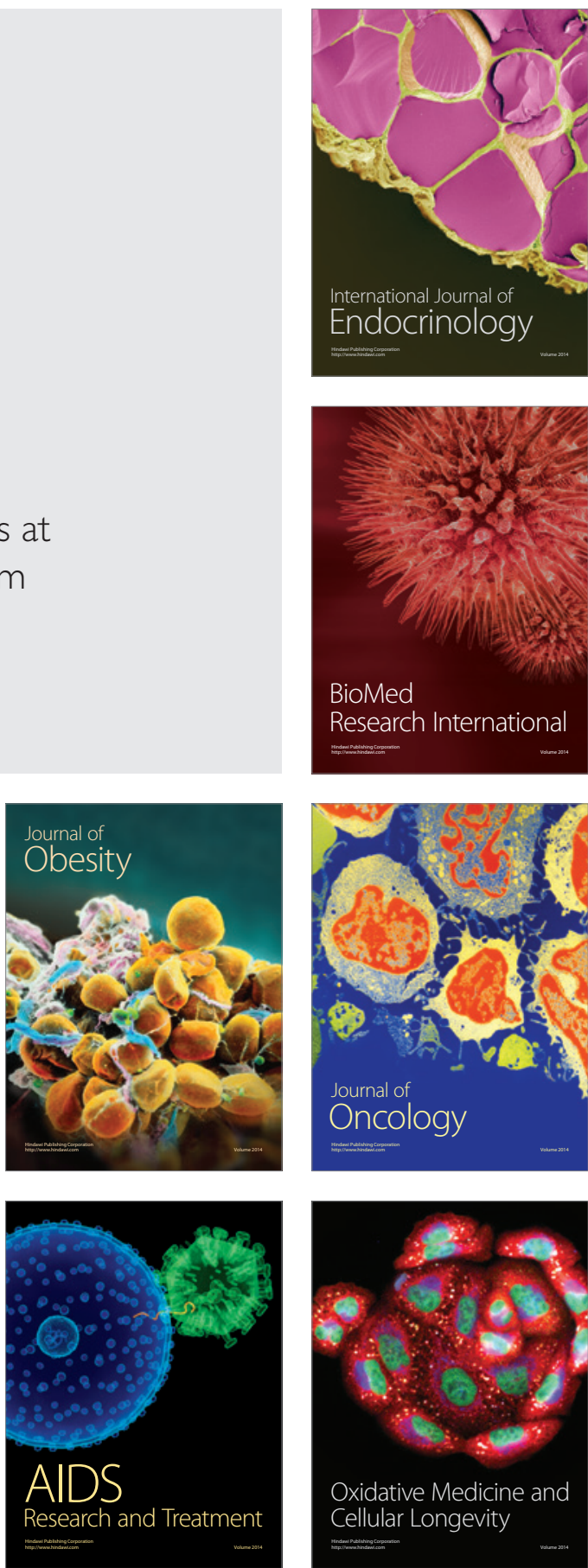